\title{
Testing SUSY GUTs at the Dark Matter and Collider Experiments
}

\author{
Cem Salih Ün, ${ }^{1}$ Zafer Alıın, ${ }^{1}$ Ali Çiçi, ${ }^{1}$, Zerrin Kırca ${ }^{1}$, Shabbar Raza ${ }^{2}$, Qaisar Shafi ${ }^{3}$, and Tug̃çe Tanımak ${ }^{1}$ \\ ${ }^{1}$ Department of Physics, Bursa Uludag̃ University, TR16059 Bursa, Turkey \\ ${ }^{2}$ Department of Physics, Federal Urdu University of Arts, Science and Technology, Karachi 75300, Pakistan \\ ${ }^{3}$ Bartol Research Intitute, Department of Physics and Astronomy, University of Delaware, Newark, DE 19716, USA
}

\begin{abstract}
We discuss low scale implications of a class of SUSY GUTs with non-universal SSB masses and confront them with the current experimental results from the direct detection of dark matter experiments, as well as the collider experiments of different center of mass energies. This class of SUSY GUTs are expected to be tested soon in direct detection dark matter experiments through the scatterings of Higgsino and Wino-like dark matters at nuclei. Besides, the stop and gluino will be able to be probed and tested up to about 5-6 $\mathrm{TeV}$ in the current and future collider experiments. These probe scales are expected to be further when high luminosities are achieved.
\end{abstract}

Keywords: Supersymmetry, Non-Universal SUSY Masses, Collider, Stop and Gluino

DOI: 10.31526/ACP.NDM-2020.15

\section{INTRODUCTION}

In this paper we discuss the low scale implications of a class of the supersymmetric grand unified theories (SUSY GUTs) with non0universal soft supersymmetry breaking (SSB) mass terms, which are represented in details in [1, 2, 3]. Non-universal SSB mass terms can be obtained by considering different sources, which break the supersymmetry (SUSY) simultaneously such as mirage mediated SUSY breaking [4], or considering F-terms, whose vacuum expectation values (VEVs) break the SUSY, belonging to non-singlet representations of the GUT gauge symmetry [5].
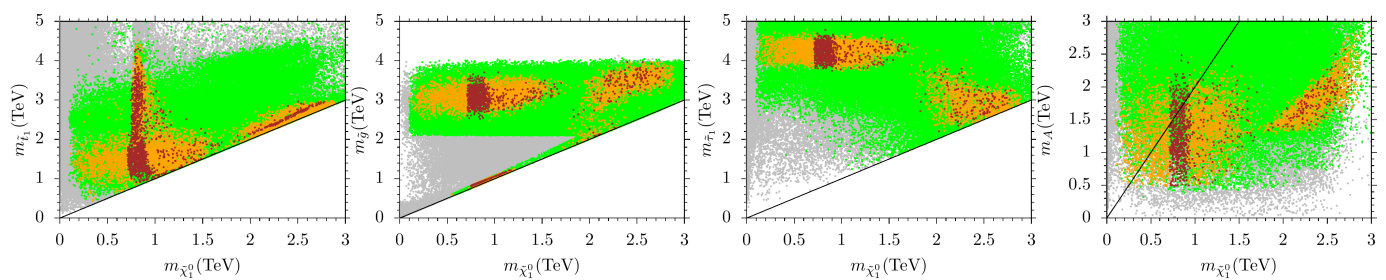

FIGURE 1: The masses of SUSY particles in comparison to the LSP neutralino. All points are compatible with the REWSB and LSP neutralino conditions. Green points satisfy the mass bounds and constraints from rare $B$-meson decays. Orange points form a subset of green, and they represent the solutions compatible with the $b-\tau$ YU of $S U(5)$ GUTs. Brown points also satisfy the WMAP bound on relic abundance of LSP neutralino within $5 \sigma$ uncertainty.

When the GUT scale boundary conditions, the low scale mass spectrum is obtained as shown in Figure 1. If one assumes the dark matter abundance is saturated by the LSP neutralino, then the results predict some coannihilation scenarios, in which the LSP neutralino co-annihilate together with stop, gluino and stau. These coannihilation processes reduce the relic abundance of the LSP neutralino into the ranges allowed by the WMAP measurements [6]. In addition, the model also predicts annihilation processes in which two LSP neutralinos annihilitate into a CP-odd Higgs boson.

The lightest mass eigenstates of stop and stau are known to be mostly the right-handed in the class of SUSY GUTs [3]; thus, the coannihilation scenarios involving the stop and stau become more effective in satisfying the constraints on the dark matter implications, when the LSP neutralino is formed mostly by MSSM higgsinos. Such solutions are also interesting in testing the dark matter implications, since their scattering cross-section at nuclei is expected to be large in comparison to Bino-like and Wino-like neutralinos.

We show the results for the spin-independent and dependent cross-sections for the dark matter scattering at nuclei in Figure 2 and compare with the current results from several direct dark matter detection experiments. As mentioned above, the Higgsinolike dark matter yields large scattering cross-section, and as can be seen from the $\sigma_{S I}-m_{\tilde{\chi}_{1}^{0}}$ plane, such solutions are below the current exclusion curve of the LUX experiment, while they are slightly above its future projection [10]. These solutions are expected to be tested soon in the experiments. There are also solutions below all the exclusion curves. These solutions predict Wino-like or Bino-Wino mixed dark matter, which predict relatively smaller scattering cross-section.

The mass spectrum shown in Figure 1 also indicates that especially stop and gluino masses are in the range that is reachable in the current and future collider experiments. We can consider the following processes in the stop analyses within the SUSY GUTs, 

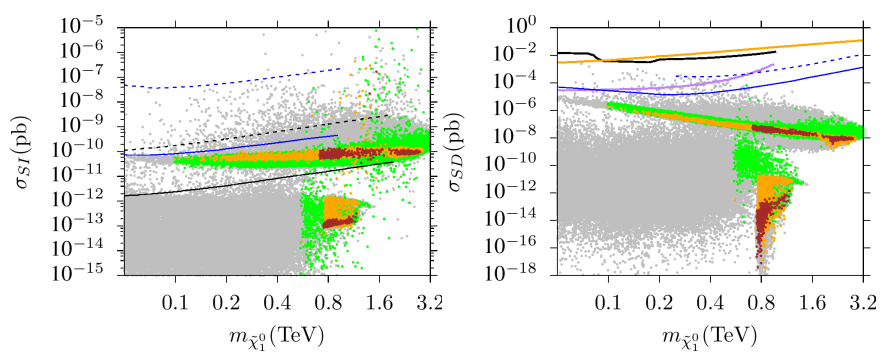

FIGURE 2: Spin-independent (left) and spin-dependent (right) scattering cross-sections versus the LSP neutralino mass. In the $\sigma_{S I}-m_{\tilde{\chi}_{1}^{0}}$ plane, the dashed (solid) blue line represents the current (future) results from the SuperCDMS experiment [7]. The dashed (solid) line indicates the current (future) results from the LUX-Zeplin experiment [8]. In the $\sigma_{S D}-m_{\tilde{\chi}_{1}^{0}}$ plane, the solid black line represents the current bound from Super-K [9], while the orange solid line is set by the LUX results [10]. The purple line is obtained from the collider analyses [11]. The dashed (solid) blue line shows the current (future) results from IceCube DeepCore.
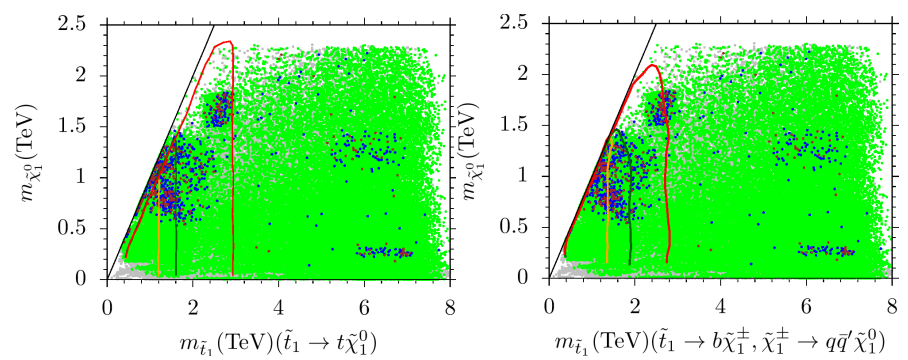

FIGURE 3: Exclusion curves for the stop mass through Signal 1 (left) and Signal 3. The green points are consistent with the current mass bounds and constraints from the rare $B$-meson decays. Blue points form a subset of green, and they are compatible with the WMAP bound, while the brown points satisfy the Planck bound on the relic abundance of LSP neutralino. In addition, orange curve represents the exclusion at the current energy, while the dark green and red are obtained at $27 \mathrm{TeV}$ and $100 \mathrm{TeV}$, repsectively. Luminosity is set to $36.1 \mathrm{fb}^{-1}$ at all energies.

$$
\begin{aligned}
& \text { Signal 1: } \quad p p \rightarrow \tilde{t}_{1} \tilde{t}_{1} \stackrel{\tilde{t}_{1} \rightarrow t \tilde{\chi}_{1}^{0}}{\longrightarrow} t \bar{t} \tilde{\chi}_{1}^{0} \tilde{\chi}_{1}^{0} \\
& \text { Signal 2: } \quad p p \rightarrow \tilde{t}_{1} \tilde{t}_{1} \stackrel{\tilde{t}_{1} \rightarrow b \tilde{\chi}_{1}^{ \pm}}{\longrightarrow} b \bar{b} \tilde{\chi}_{1}^{ \pm} \tilde{\chi}_{1}^{\mp} \stackrel{\tilde{\chi}_{1}^{ \pm} \rightarrow W^{ \pm} \tilde{\chi}_{1}^{0}}{\longrightarrow} \rightarrow b \bar{b} W^{ \pm} W^{\mp} \tilde{\chi}_{1}^{0} \tilde{\chi}_{1}^{0} \\
& \text { Signal 3: } \quad p p \rightarrow \tilde{t}_{1} \tilde{t}_{1} \stackrel{\tilde{t}_{1} \rightarrow b \tilde{\chi}_{1}^{ \pm}}{\longrightarrow} b \bar{b} \tilde{\chi}_{1}^{ \pm} \tilde{\chi}_{1}^{\mp} \stackrel{\tilde{\chi}_{1}^{ \pm} \rightarrow q \bar{q}^{\prime} \tilde{\chi}_{1}^{0}}{\longrightarrow} \rightarrow b \bar{b}\left(q \bar{q}^{\prime}\right)\left(q \bar{q}^{\prime}\right) \tilde{\chi}_{1}^{0} .
\end{aligned}
$$

While the processes referred to Signal 1 and Signal 3 can provide sensitive results in the current and future experiments, Signal 2 is not available for the current experiments. In order to have chargino decaying into a LSP neutralino along with a $W-$ boson, it has to be Wino-like chargino, while LSP neutralino is to be Bino-like. However, since the lightest stop mass eigenstate is found to be mostly right-handed, the Wino-like chargino interacts with it quite weakly, proportional to the mixing of the left-handed stop in the lightest stop mass eigenstate. However, it can provide some sensitive results in the future experiments with higher center of mass energy and luminosity [3].

Following similar to the experimental analyses [12]; the exclusion curves, which can be probed in the current and future collider experiments, can be obtained as shown in Figure 3. According to our results, the current experiments can probe the stop mass up to about $1.2 \mathrm{TeV}$, while it can be probed up to about $1.8 \mathrm{TeV}$ and $3 \mathrm{TeV}$ in the future colider experiments of $27 \mathrm{TeV}$ and $100 \mathrm{TeV}$ center of mass energy, respectively.

Similar analyses can be also followed for the gluino. The current experimental mass bounds on the gluino is about 2.1 TeV [12], while it reduces to $m_{\tilde{g}} \geq 800 \mathrm{GeV}$, when it is next to LSP (NLSP). Considering the results shown in Figure 1, the gluino is realized mostly heavier than the stop, and one can also add another decay channel in which a gluino decays into a stop quark along with a top quark. If we follow similar analyses, which are performed in the experimental analyses over the gluino mass. We summarize our findings for the exclusion curves on the gluino mass in Figure 4. While The current experiments have enough sensitivity to test the gluino mass up to about $2 \mathrm{TeV}$, the future collider experiments will be able to probe its mass up to about $3 \mathrm{TeV}$ and $6 \mathrm{TeV}$, when the center of mass energy is set to $27 \mathrm{TeV}$ and $100 \mathrm{TeV}$ respectively. This decay channel of gluino can also indirectly probe the stop mass as shown in the right panel of Figure 4. It can lift the mass bound on the stop up to about $1.8 \mathrm{TeV}$ un the current experiments (orange curve), while the stop can be probed up to about 2.2 and $4.4 \mathrm{TeV}$ in the collider experiments with the center of mass energy of $27 \mathrm{TeV}$ and $100 \mathrm{TeV}$, respectively. Note that despite being indirect bounds, the $\tilde{g} \rightarrow \tilde{t} t$ decay channel may provide more sensitive results on the stop mass. 

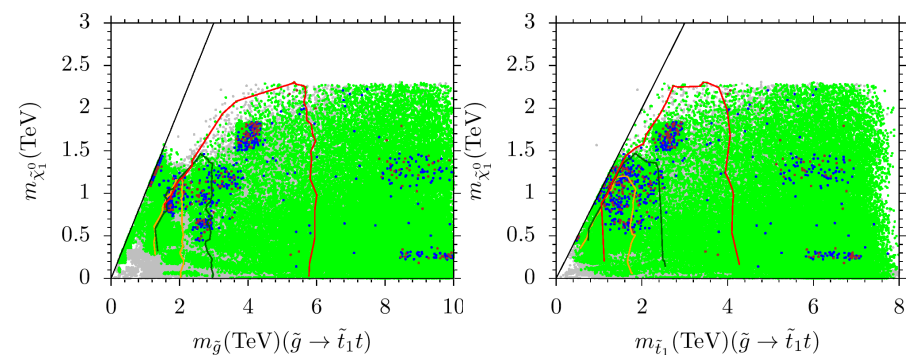

FIGURE 4: Summary of our findings in the $m_{\tilde{\chi}_{1}^{0}}-m_{\tilde{g}}$ and $m_{\tilde{\chi}_{1}^{0}}-m_{\tilde{t}_{1}}$ planes. The color coding is the same as in Figure 3 . The orange curve represents the exclusion at $14 \mathrm{TeV}$, while the dark green and the red curves are obtained for $27 \mathrm{TeV}$ and $100 \mathrm{TeV}$ respectively.

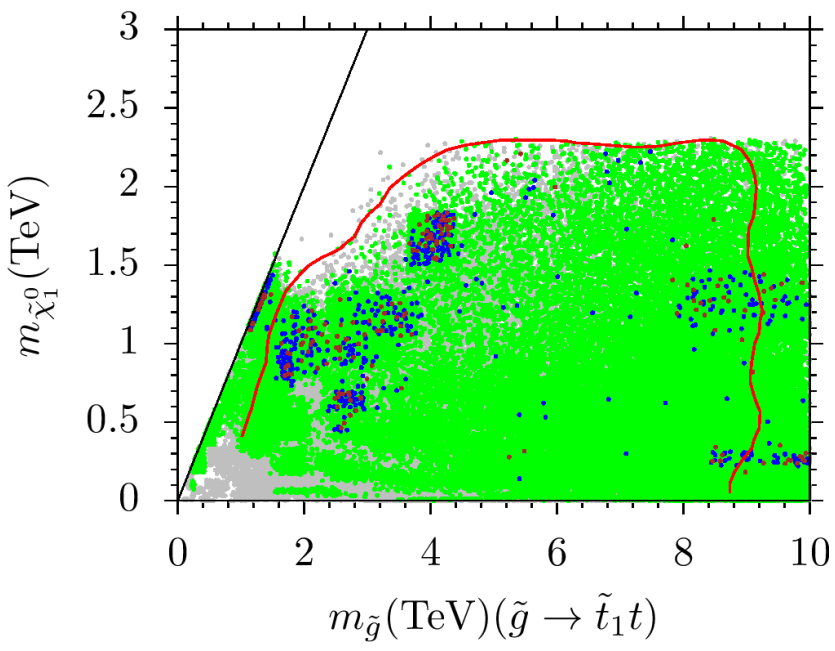

FIGURE 5: Gluino probe at the $100 \mathrm{TeV}$ center of mass energy and $3000 \mathrm{fb}^{-1}$ Luminosity. The color coding is the same in Figure 3. The red curve represents the gluino reach when the luminosity is set to $3000 \mathrm{fb}^{-1}$.

Before concluding, we also consider the gluino mass probe in the collider experiments with $100 \mathrm{TeV}$ center of mass energy and $3000 \mathrm{fb}^{-1}$ luminosity. our analyses over the $\tilde{g} \rightarrow \tilde{t} t$ within the class of SUSY GUTs reveal that the gluino mass can be probe up to about $9 \mathrm{TeV}$ when a high luminosity is achieved in the future collider experiments with the center of mass energy of $100 \mathrm{TeV}$.

\section{CONCLUSION}

We discussed the dark matter implications and probe of the low scale mass spectrum through the decay modes of the stop and gluino within a class of SUSY GUTs with non-universal SSB mass terms implemented at the GUT scale. This class of models are expected to be tested in the current and near future direct detection dark matter experiments via the scattering of Higgsino and Wino-like LSP neutralinos at nuclei. In addition, the stop and gluino particles will be available in the current and near future collider experiments. The stop can be tested up to $1.2 \mathrm{TeV}$ in the current experiments, while the future experiments will be sensitive enough to probe its mass up to about $3 \mathrm{TeV}$. Similarly, the gluino mass can be tested up to about $2 \mathrm{TeV}$ in the current experiments, while it will be able to be probed up to about $6 \mathrm{TeV}$ in the future experiments. These bounds and probe scales are expected to be further, when the high luminosity is achieved.

\section{ACKNOWLEDGEMENTS}

We would like to thank the organizing committee of NDM 2020 for having such a nice meeting and their hospitality. The work of CSÜ, ZA, A.Ç, ZK and TT are supported by the Scientific and Technological Research Council of Turkey (TUBITAK) Grant no. MFAG-118F090. Q. S. is supported in part by DOE under Grant no. DE-SC 0013880.

\section{References}

[1] S. Raza, Q. Shafi and C. S. Un, JHEP 05, 046 (2019) doi:10.1007/JHEP05(2019)046 [arXiv:1812.10128 [hep-ph]].

[2] Z. Altın, A. Ç. Içi, Z. Kırca, Q. Shafi and C. Salihün, [arXiv:1910.01457 [hep-ph]].

[3] Z. Altın, Z. Kırca, T. Tanımak and C. Salih Ün, [arXiv:1910.12992 [hep-ph]].

[4] K. Choi, K. S. Jeong and K. i. Okumura, JHEP 0509, 039 (2005); [hep-ph/0504037]. K. Choi and H. P. Nilles, JHEP 0704, 006 (2007) [hepph/0702146 [HEP-PH]]; T. Kobayashi, arXiv:1504.05634 [hep-ph]; A. Anandakrishnan and S. Raby, Phys. Rev. Lett. 111, no. 21, 211801 (2013) 
[arXiv:1303.5125 [hep-ph]]; H. Baer, V. Barger, H. Serce and X. Tata, Phys. Rev. D 94, no. 11, 115017 (2016) [arXiv:1610.06205 [hep-ph]].

[5] S. P. Martin, Phys. Rev. D 79, 095019 (2009) doi:10.1103/PhysRevD.79.095019 [arXiv:0903.3568 [hep-ph]], and references therein.

[6] G. Hinshaw et al. [WMAP], Astrophys. J. Suppl. 208, 19 (2013) doi:10.1088/0067-0049/208/2/19 [arXiv:1212.5226 [astro-ph.CO]].

[7] P. L. Brink et al. [CDMS-II Collaboration], eConf C 041213, 2529 (2004) [astro-ph/0503583]

[8] D. S. Akerib et al. [LUX-ZEPLIN Collaboration], arXiv:1802.06039 [astro-ph.IM].

[9] T. Tanaka et al. [Super-Kamiokande Collaboration], Astrophys. J. 742, 78 (2011) doi:10.1088/0004-637X/742/2/78 [arXiv:1108.3384 [astroph.HE]].

[10] D. S. Akerib et al. [LUX Collaboration], Phys. Rev. Lett. 116, no. 16, 161302 (2016) doi:10.1103/PhysRevLett.116.161302 [arXiv:1602.03489 [hepex]].

[11] V. Khachatryan et al. [CMS Collaboration], Eur. Phys. J. C 75, no. 5, 235 (2015) doi:10.1140/epjc/s10052-015-3451-4 [arXiv:1408.3583 [hep-ex]].

[12] T. A. Vami [ATLAS and CMS Collaborations], arXiv:1909.11753 [hep-ex]; J. Mitrevski [ATLAS Collaboration], PoS DIS 2018, 079 (2018); C. Collard [CMS Collaboration], arXiv:1709.00868 [hep-ex]; The ATLAS collaboration [ATLAS Collaboration], ATLAS-CONF-2016-077. 